\title{
Co-design of Color Identification Applications Using Scenario-Based Personas for People with Impaired Color Vision
}

\author{
Mawaddah AlSabban ${ }^{1(凶)}$, Arwa Karim² ${ }^{2}$ Virginia H. Sun ${ }^{3}$, Jood Hashim ${ }^{4}$, \\ and Osama AlSayed ${ }^{4}$ \\ ${ }^{1}$ Saudi Authority for Intellectual Property, Riyadh, Saudi Arabia \\ Msabban@saip.gov.sa \\ ${ }^{2}$ King Abdullah University of Science and Technology, Thuwal, Saudi Arabia \\ arw2.ak.23@gmail.com \\ 3 Massachusetts Institute of Technology, Cambridge, MA, USA \\ gsun@mit. edu \\ 4 CoCreate Fellowship for the Humanistic Co-design of Access Technology, Riyadh, \\ Saudi Arabia \\ joodayh@gmail.com, osamab.alsayedegmail.com
}

\begin{abstract}
Designers and system analysts have long struggled to extract and repurpose data from user research by laboriously presenting content in the form of storyboards, behavioral-type personas, or journey maps. An alternative is to convey insights from user research through scenario-based personas that represent user research data through storytelling. This provides a more streamlined way to convey data rather than character-based personas; however, scenarios are effortful for developers to articulate and envision. In this work, we empower assistive technology development teams to access authentic user experiences with scenario-based personas through tangible and digital artifacts. Scenario-based personas were used for conveying the results of a user analysis study for color identification mobile applications for people with visual impairments. We developed scenario-based personas for persons with impaired color vision based on the main contexts identified in user research studies. The method combines personas depicted in silhouettes of people with impaired color vision and scenario contexts that capture the place and activities. Silhouettes were used in the artifacts to reduce the bias that a face often generates in persona-based scenarios. Preliminary findings suggest that scenario-based persona tools were effective in describing the stories and context behind why a person with a visual disability would consider a color identification application. Through this method, scenario-based personas were able to foster understanding of the application's target user population by showing their main contexts of using these mobile applications and create playful and tangible artifacts to capture and convey such user information to designers and developers in the Humanistic Co-Design community. Methodological considerations for using scenario-based personas in user research will be discussed.
\end{abstract}

Keywords: Participatory design · Mobile applications · Co-design ·

Co-creation $\cdot$ Color identification $\cdot$ Scenarios $\cdot$ Visually impaired 


\section{Introduction}

Personas are well established in the user-centered design (UCD) and co-design contexts; however, they are often not utilized by developers of assistive technology [1-3]. Scenarios are critical both for designing assistive technologies and for usability testing with persons with disabilities. Moreover, personas depicting fictional characters with photos are often perceived differently when the selected characters do not reflect the diversity of a population. Different variations of personas and scenarios have been reported in the HCI literature and explored in practice $[1,2]$. The research on personas and scenarios in the design of assistive technology is less explored and inadequately understood [2].

Many people who have visual disabilities worldwide depend on mobile applications to augment their sensory abilities [4-8]. A large part of them are unable to detect colors. In recent years, color identification technologies have been designed for people who have visual disabilities [6, 7]. Techniques range from color detection based on image processing (e.g. Seeing AI [9]) to fiducial marker-based methods [10, 11].

The design process for innovative products is often more effective when users are involved as co-designers. Towards this model of co-design, personas and scenarios emerge as important tools for communicating the needs and requirements that would be considered in the product designs [3]. While current applications can partially assist the visually impaired, they lack features that could prove useful to the user. Not using personas as a foundation for building an application could be considered a reason and factor for the lack of helpful features. Personas are an essential part of the design phase for an application, as they represent the needs of users and how they plan to use an application [12].

In designing color identification applications, it is important for developers to focus on the requirements of users who seek mobile applications to translate images captured by the camera into spoken words to aid them in identifying the color. The spoken words are extracted from a color dictionary which is often accessible for mainstream languages, yet is not available for some languages, such as Arabic. Understanding the diverse users, different contexts, and scenarios of usage requires collective efforts of users and designers.

This paper is structured as follows: Sect. 2 describes the background and motivation for using personas and scenarios in user-centered design for assistive technology. Following that, we describe the method in Sect. 3 followed by the design implications in Sect. 4. We conclude in Sect. 5 with a summary of contributions and directions for future research.

\section{Personas and Scenarios in Designing Assistive Technology for People with Visual Impairments}

Although mobile devices include accessibility features, individuals who have visual impairments still face a variety of challenges such as small form factors, small or undifferentiated keys, and tiny on-screen text [13]. Personas and scenarios arise as important 
tools for communicating the needs and requirements that would be considered in the design of the product. Towards the overarching goal of accessible mobile application design, we discuss here existing scenario-based personas and applications for individuals with visual impairments.

\subsection{Scenarios in Assistive Technology Design}

Designers of assistive technologies often inadvertently propose solutions based on assumptions that are not grounded in user-research [14]. Some assistive technologies require far too much scrolling or directional swipes to produce actions on a mobile screen. Furthermore, people who have lost some sensitivity in their fingers or those who have visual impairments would benefit from better-designed buttons and switches [15].

Mobile accessibility has plagued the assistive technology field for many years [16]. In order to solve this issue, designers have utilized personas and scenarios to give life and substance to user research findings.

Personas have been employed in assistive technology in various ways, one of them being the AEGIS project (Accessibility Everywhere: Groundwork, Infrastructure, Standards). "The AEGIS project attempts to establish whether 3rd generation access techniques would provide a more accessible, more exploitable, and deeply embeddable approach to mainstream information and communication technology (ICT)" [17]. AEGIS identifies user needs and interaction models for several user groups and develops open source-based generalized accessibility support for mainstream ICT devices/applications [17]. Within the AEGIS project, a variety of methods were used to identify users' needs. A total of seventeen personas were made and were presented across five sections. Each persona is introduced with a name, a photo, and a short description of his personal situation. Furthermore, an overview of the problems of a persona in using the technology in itself or in using the assistive technology is provided. Based on these problems, the next section of each persona gives an overview of their needs and wants [18].

As a result of their approach, the researchers ensured that the needs of a number of user groups were considered in all cycles of design and development of accessible applications. The user groups include users with visual, hearing, movement, speech, and cognitive impairments, in addition to the application developers themselves.

In another context, personas were also used to exemplify the process of using mobile applications by an aging patient population. The resultant personas improved the design, development, and implementation plans of a smartphone application to assist chronically ill aging Chinese diabetic population capable of disease self-management [19].

\subsection{Personas in Assistive Technology Design for the Blind and/or Low-Vision}

The utilization of personas has become an essential tool that assists designers, developers, and the assistive technology industry. Using personas that simulate the needs of persons who are blind and/or low-visioned can help with understanding the needs of the visually impaired which in-turn leads to the development of assistive technologies. 
To better understand the needs of persons who are blind and/or low-visioned, in 2018, designers E. Brule and C. Jouffrais have used scenario-based personas to include children living with visual impairments in the design process. They used activities aimed at raising empathy through storytelling, persona cards, and role-playing. Their study aimed at better understanding the educational context and the experiences of visually impaired children [20].

The field study helped to better describe children living with visual impairment and how they interact with assistive technologies. It also demonstrated how those interactions are shaped by a larger context (e.g. policy, culture, etc.). These observations guided the development of a set of design cards and workshop activities, which aimed to improve the representation of users and increase empathy [20].

\section{Method}

In co-design models, scenarios are often used to understand the needs of the user for the product, service, and/or system in a specific context [7]. The work on personas in this study stemmed from the need to find ways to bring co-designers, engineers, and developers together into a single, cohesive team. The goal of the scenario-based personas was to allow the development team to live and breathe the target user population's world in the context of color identification scenarios.

\subsection{Designing Scenario-Based Personas}

The process of designing the scenario-based personas started with semi-structured interviews as part of the user research in a Humanistic Co-design for assistive technology workshop. From our ethnographic research on people with visual impairments and impaired color vision, we focused our personas in the first iteration of design on the user mode of action rather than the user demographics (such as age, gender, abilities) or their essence (e.g. attitudes) as depicted in Fig. 1.
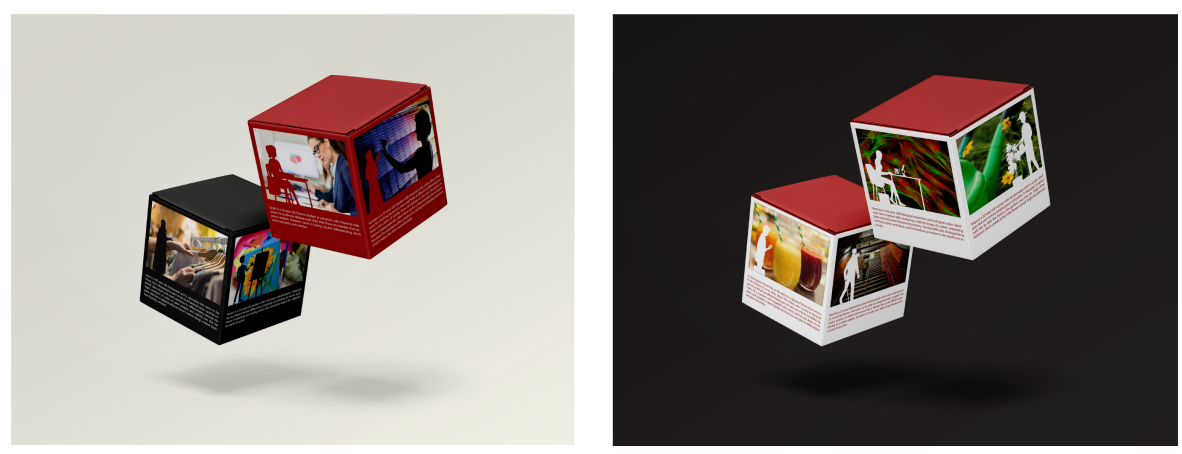

Fig. 1. Tangible scenario-based persona artefacts for designers and co-designers 
Designing scenarios involved identifying the different contexts of use in which we envisioned color identification would take place, such as in the context of grocery shopping, clothing shopping, paint color selection, or vehicle showroom. Following that, silhouettes are overlaid on the backdrop image as in Fig. 2.

The scenario depicted in Fig. 2 could apply to fruits that are relatively similar in shape and form such as apples, grapes, and pears but vary in color, flavor, and nutrition. Color identification is important for shoppers when buying apples, to facilitate choosing those without any bruises or soft, mushy spots and to look for fruit with shiny skin—dull skin

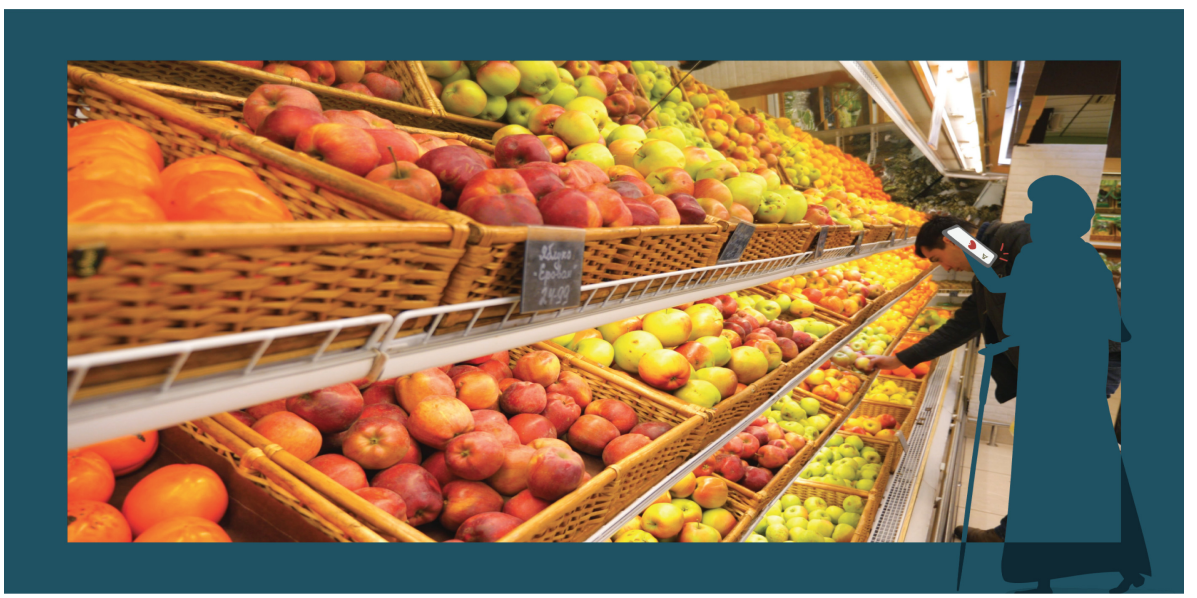

Fig. 2. Silhouette of a shopper would be overlaid on a "selecting fruit based on color" activity in a grocery store setting.

Visual scene depicting objects in a restaurant Description of people, place, and issue(s)

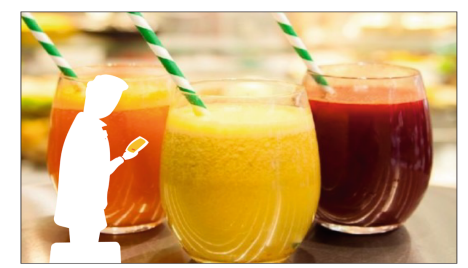

A server places the drinks on the table for a group of diners then returns glasses and had no differentiating garnish and can only be identified by color. Ali is a person with impaired color vision, and he was alone at by between the drinks.

\section{Scenario in a restaurant}

A server places the drinks on the table for a group of diners then returns to the kitchen to serve other diners. The drinks were served in identical glasses and had no differentiating garnish and can only be identified by color.

Ali is a person with impaired color vision, and he was alone at the table waiting for his friends to join, and he wanted to select his drink but was not able to differentiate between the drinks.

Fig. 3. Silhouette of a diner overlaid on a 'restaurant table' scene 
hints at a lack of crispness and flavor. Independent detection of these subtle variations in color empower users in the activity of shopping for these items and in daily living when these items are selected for meals or during cooking (Figs. 3, 4, 5, 6, 7, 8, 9, 10, 11, 12 and 13).

Visual scene of shopping in a clothing store

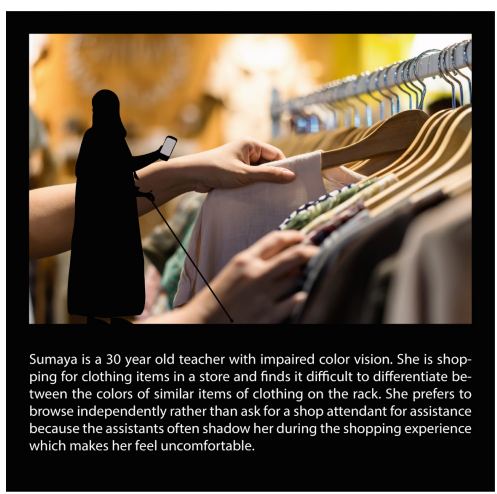

Description of people, place, and issue(s)

\section{Scenario in a clothing store}

Sumaya is a 30 -year-old teacher with impaired color vision. She is shopping for clothing items in a store and finds it difficult to differentiate between the colors of similar items of clothing on the rack.

Sumaya prefers to browse independently rather than ask for a shop attendant for assistance because the assistants often shadow her during the shopping experience which makes her feel uncomfortable.

Fig. 4. Silhouette of a shopper overlaid on a clothing shop scene with a shopper browsing items

Visual scene of a customer in a paint store

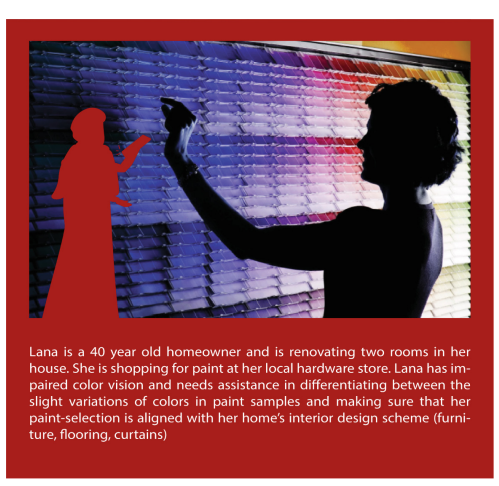

Description of people, place, and issue(s)

\section{Scenario in a paint store}

Lana is a 40-year-old homeowner and is renovating two rooms in her house. She is shopping for paint at her local hardware store. Lana has impaired color vision and needs assistance in differentiating between the slight variations of colors in paint samples and making sure that her paint-selection is aligned with her home's interior design scheme (furniture, flooring, curtains)

Fig. 5. Silhouette of a shopper overlaid over wall displays of a paint shop 
Visual scene of a car showroom

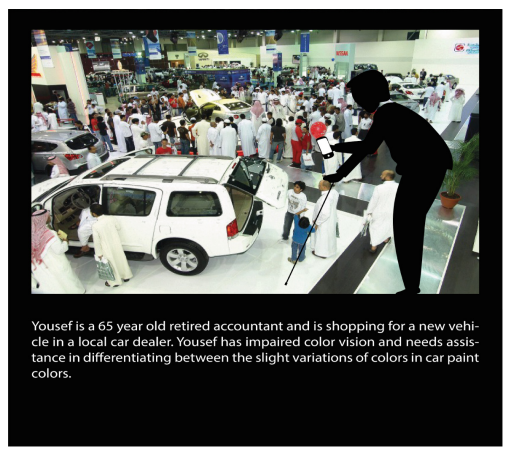

Description of people, place, and issue(s)

\section{Scenario in a car showroom}

Yousef is a 65-year-old retired accountant and is shopping for a new vehicle in a local car dealer. Yousef has impaired color vision and needs assistance in differentiating between the slight variations of colors in car paint colors.

Fig. 6. Silhouette of a shopper overlaid on a crowded scene in a showroom or exhibit

Visual scene of an art studio

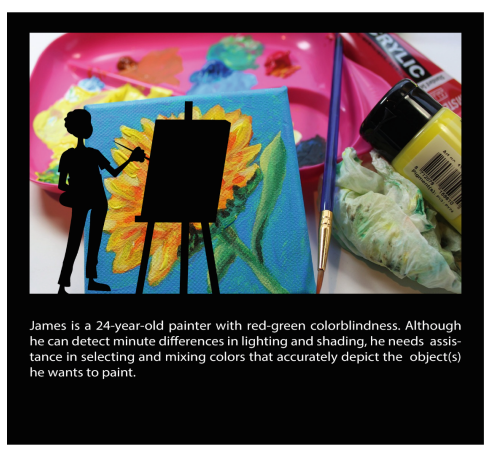

Description of people, place, and issue(s)

\section{Scenario in an art studio}

James is a 24-year-old painter with red-green colorblindness. Although he can detect minute differences in lighting and shading, he needs assistance in selecting and mixing colors that accurately depict the object(s) he wants to paint.

Fig. 7. Silhouette of a painter overlaid in an art studio

Visual scene of gift-wrapping station

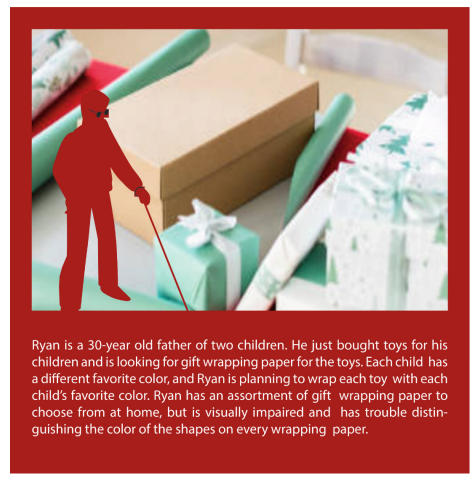

Description of people, place, and issue(s)

\section{Scenario in a gift-wrapping station}

Ryan is a 30 -year old father of two children. He just bought toys for his children and is looking for gift wrapping paper for the toys. Each child has a different favorite color, and Ryan is planning to wrap each toy with each child's favorite color. Ryan has an assortment of gift-wrapping paper to choose from at home, but has a visual impairment and has trouble distinguishing the color of the shapes on every wrapping paper.

Fig. 8. Silhouette of a shopper overlaid in a gift-wrapping station 
Visual scene of taking photos in a studio

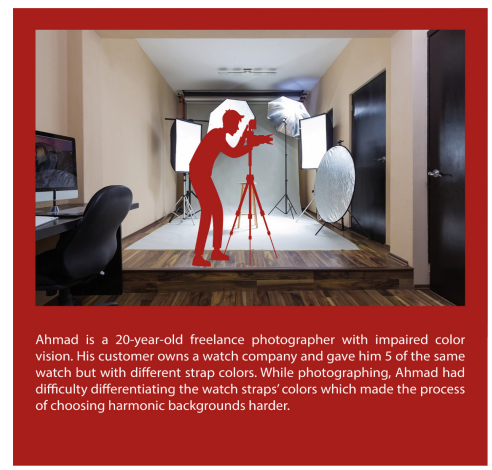

Description of people, place, and issue(s)

\section{Scenario in a photography studio}

Ahmad is a 20-year-old freelance photographer with impaired color vision. His customer owns a watch company and gave him five of the same watch but with different strap colors. While photographing, Ahmad had difficulty differentiating the watch straps' colors which made the process of choosing harmonic backgrounds harder.

Fig. 9. Silhouette of a photographer overlaid on a photography studio scene with a photographer browsing different backgrounds

Visual scene of a student in a finance class

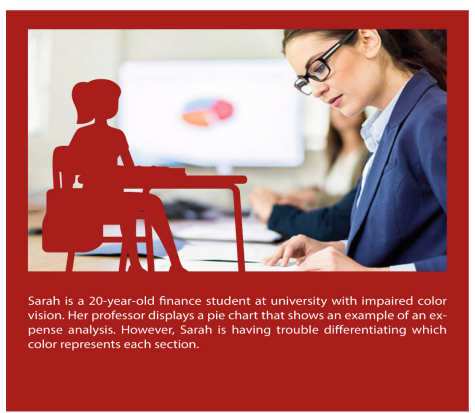

Description of people, place, and issue(s)

\section{Scenario in a university class}

Sarah is a 20-year-old finance student at university with impaired color vision. Her professor displays a pie chart that shows an example of an expense analysis. However, Sarah is having trouble differentiating which color represents each section.

Fig. 10. Silhouette of a student overlaid on a finance class with a pie chart

Visual scene of an adult in a metro station.

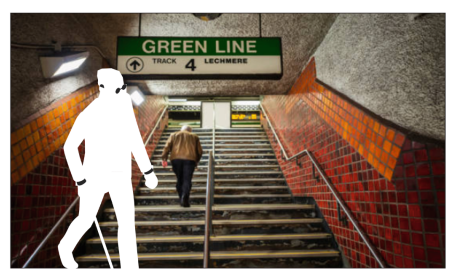

David is a 32-year-old professional with impaired color vision who recently moved to Boston. He wants to be able to independently navigate the ferring at a metro station, he wants to make sure that he has located the correct metro line.
Description of people, place, and issue(s)

\section{Scenario in a metro station}

David is a 32-year-old professional with impaired color vision who recently moved to Boston. He wants to be able to independently navigate the metro system, in which each metro line is labeled by a color. While transferring at a metro station, he wants to make sure that he has located the correct metro line.

Fig. 11. Silhouette of an adult overlaid on a metro station 
Visual scene of a researcher analyzing an image

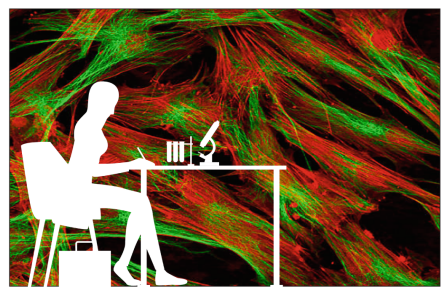

Vanessa is a 36-year-old biological researcher with red-green color blindness. She is tasked with analyzing a stained image of a plant expressing red from green and black, and is therefore uncertain if her results are accurate.
Description of people, place, and issue(s)

\section{Scenario in research lab}

Vanessa is a 36-year-old biological researcher with red-green color blindness. She is tasked with analyzing a stained image of a tissue sample expressing both red and green-fluorescent proteins. She has difficulty distinguishing red from green and black, and is therefore uncertain if her results are accurate.

Fig. 12. Silhouette of a researcher analyzing a fluorescent-stained image

Visual scene of an adult in a garden

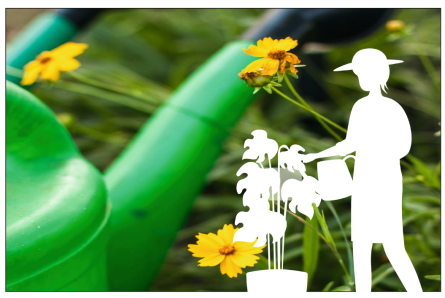

Maya is a 22-year-old woman with deuteranopes which is a color blindness that can confuse bright greens with yellow. A shop asked to partne with her to take the flowers she gardens once they are bright yellow
However, she is unable to tell if the flowers turned bright and yellow yet.

\section{Description of people, place, and issue(s)}

\section{Scenario in a garden}

Maya is a 22-year-old woman with deuteranopes, a color blindness that can confuse bright greens with yellow. A shop asked to partner with her to take the flowers she gardens once they are bright yellow. However, she is unable to tell if the flowers turned bright and yellow yet.

Fig. 13. Silhouette of a gardener tending to yellow flowers in a garden. (Color figure online)

\section{Discussion and Design Implications}

While senses such as sound, taste, smell, and touch can be used to either substitute or supplement low vision in certain scenarios, color is one of the unique properties that can only be identified through vision. The above personas were created through user discussions and case studies of individuals with various vision impairments, including color-blindness, low vision, and complete blindness. Depending on each individual's unique interests and abilities, the user may present different scenarios in which they would use a color-identification application. Additional user-scenarios can be created by recruiting more co-designers and/or exploring situations in which they would need accurate color detection and identification to perform a task. In this section, we describe 
the design implications of such scenario-based personas by outlining its use cases and impact.

\subsection{Use Cases of Scenario-Based Personas for People with Impaired Color Vision}

In designing assistive technology, it is important for the designer to understand the situation in which such technology would be used. Although it is recommended to have people with visual impairments to play an active role in designing assistive technology as a co-designer [21], scenario-based personas can be used in place of a co-designer if unavailable. Assistive technology hackathons, for example, have recently grown in popularity and draws in both students and professionals around the world [22-24]. These user-centered hackathons require the designers to understand the context and perspective surrounding the individual and his/her problems [25]. One or more of the scenarios outlined in Sect. 3 may be used as a problem statement, or launching board, for a hackathon project. The resulting solution may then be adopted by people with impaired color vision and applied in real-world situations.

The aforementioned scenario-based personas may also be used in educational settings, such as design classes and assistive-technology workshops. In an example usecase scenario, a student in a design class may choose a persona to expand on. While conducting research on the selected scenario, the designer may assess whether existing color-identification applications adequately address the needs of the individual described in the persona. After uncovering issues with the existing solutions, the designer can then create a prototype that will allow the individual to independently navigate through the described scenario. If time permits, further designing, testing, and iterating can lead to a fully-functional product. Through this activity, the designer will have conducted research, defined a problem, generated ideas, developed designs, and evaluated designs - all principal components of the user-centered design process [26].

Even when co-designers are available, the scenario-based personas we described can help supplement their input when designing a color-identification tool. By designing an application based on multiple users and personas, the designer can ensure that the end-result is scalable and applicable across a wider range of scenarios.

\subsection{Impact of Scenario-Based Personas for People with Impaired Color Vision}

By encouraging the use of scenario-based personas when developing assistive technology for people with impaired color vision, the designer approaches the project by considering the individual, activity, and context for which the technology is to be applied to. This framework, known as the human activity assistive technology (HAAT) model, is necessary to consider when designing and implementing assistive technology [27].

Through these scenarios, the designer may also adopt an interactional model of color vision impairment, rather than a functional-limitation model. That is, rather than attributing a person's disabilities for any disadvantages, the interactional model states that the interaction between the environment and the individual determines if a disability exists [28]. In these scenarios, a properly designed color-identification application will prevent people with impaired color vision from being disadvantaged at all. Adopting an interactional model may allow individuals to approach disability with a more positive 
perception by acknowledging that disadvantages may stem from society's treatment of people with disabilities, rather than the disability itself.

These scenario-based personas may introduce individuals to personal stories that are outside of their own experience. Recognizing the unique characteristics, abilities, and interests associated with the scenario-based personas may help designers develop greater empathy towards people with impaired color vision. The personas may also help inspire designers by providing a glimpse into what users they want to interview and recruit in developing a product. With the development of an application that fits the co-designer's specific needs, the co-designer would feel more empowered as they can navigate through these situations without the assistance of another individual.

\section{Conclusion and Future Work}

\subsection{Conclusion}

Although developers and designers try to keep the needs of users in mind, the design of the application suggests that the development process may be driven by assumptions of the user [2]. We found that scenario-based personas present as a valid option for designers in identifying a user's specific needs. Scenario-based personas introduce individuals to personal stories that are outside of their own experience, which in-turn helps the designers develop more efficient assistive technologies. We believe that the scenariobased personas we developed through user interviews and case studies will be able to foster understanding of the application's target user population by offering various contexts for using color-identification applications.

\subsection{Future Work}

To develop a more accurate understanding of the various use-cases for coloridentification applications, more persona-based scenarios should be collected. Additional scenarios may be discovered via interviews of individuals with color vision impairments and their caregivers (i.e. family, personal care assistants, ophthalmologists).

As demonstrated in Fig. 1, the scenario-based personas can be developed into tangible objects that can be used for additional research (e.g. via persona box design). We hope to further study how such objects can be integrated into design settings to increase empathy for people with visual impairments and encourage principles of universal design. An example experimental design could consist of several groups of design students, each given a subset of persona-based scenarios displayed on a tangible object, with a control group given no personas. After assigning each group with an identical design task, the final design could be evaluated based on accessibility. Similarly, we hope to further analyze where and when persona-based scenarios can be used to substitute and/or supplement co-designers with visual impairments, and if one result leads to better design outcomes and greater empathy over the other.

Acknowledgment. We thank the Humanistic Co-Design Initiative and the Human-Computer Interaction (HCI) Lab for supporting this work. We also thank the Saudi Authority for Intellectual Property (SAIP) and the Saudi Health Council's National Lab for Emerging Health Technologies 
for hosting and mentoring. This work is part of the authors' project that is carried out under the CoCreate Fellowship for Humanistic Co-Design of Access Technologies. We would also like to thank the participants in the user study, as well as Dr. Areej Al-Wabil, Dr. Shiroq Al-Megren, Dr. Kyle Keane, and Anna Musser, for their mentorship and support.

\section{References}

1. Saez, A.V., Domingo, M.G.G.: Scenario-based persona: introducing personas through their main contexts. In: Extended Abstracts on Human Factors in Computing Systems (CHI EA 2011), p. 505. Association for Computing Machinery, New York (2011). https://doi.org/10. 1145/1979742.1979563

2. Moser, C., Fuchsberger, V., Neureiter, K., Sellner, W., Tscheligi, M.: Revisiting personas: the making-of for special user groups. In: CHI '12 Extended Abstracts on Human Factors in Computing Systems (CHI EA 2012), pp. 453-468. Association for Computing Machinery, New York (2012). https://doi.org/10.1145/2212776.2212822

3. Chang, Y.N., Lim, Y.K., Stolterman, E.: Personas: from theory to practices. In: Proceedings of the 5th Nordic conference on Human-computer interaction: building bridges (NordiCHI 2008), pp. 439-442. Association for Computing Machinery, New York (2008). https://doi. org/10.1145/1463160.1463214

4. Medeiros, A.J., Stearns, L., Findlater, L., Chen, C., Froehlich, J.E.: Recognizing clothing colors and visual textures using a finger-mounted camera: an initial investigation. In: Proceedings of the 19th International ACM SIGACCESS Conference on Computers and Accessibility (ASSETS 2017), pp. 393-394. Association for Computing Machinery, New York (2017). https://doi.org/10.1145/3132525.3134805

5. Nguyen, R., Geddes, C.: Exploring haptic colour identification aids. In: The 21st International ACM SIGACCESS Conference on Computers and Accessibility (ASSETS 2019), pp. 709711. Association for Computing Machinery, New York (2019). https://doi.org/10.1145/330 8561.3356111

6. Flatla, D.R., Andrade, A.R., Teviotdale, R.D., Knowles, D.L., Steward, C.: ColourID: improving colour identification for people with impaired colour vision. In: Proceedings of the 33rd Annual ACM Conference on Human Factors in Computing Systems (CHI 2015), pp. 35433552. Association for Computing Machinery, New York (2015). https://doi.org/10.1145/270 2123.2702578

7. Li, W., Flatla, D.R.: 30 years later: has CVD research changed the world? In: The 21 st International ACM SIGACCESS Conference on Computers and Accessibility (ASSETS 2019), pp. 584-590. Association for Computing Machinery, New York (2019). https://doi.org/10. $1145 / 3308561.3354612$

8. Popleteev, A., Louveton, N., McCall, R.: Colorizer: smart glasses aid for the colorblind. In: Proceedings of the 2015 workshop on Wearable Systems and Applications (WearSys 2015), pp. 7-8. Association for Computing Machinery, New York (2015). https://doi.org/10.1145/ 2753509.2753516

9. Seeing AI App from Microsoft. https://www.microsoft.com/en-us/ai/seeing-ai. Accessed 18 June 2020

10. Mattos, A.B., Cardonha, C., Gallo, D., Avegliano, P., Herrmann, R., Borger, S.: Marker-based image recognition of dynamic content for the visually impaired. In: Proceedings of the 11th Web for All Conference, pp. 1-4. Association for Computing Machinery, New York (2014). https://doi.org/10.1145/2596695.2596707

11. Prasanna, S., Priyadharshini, N., Pugazhendhi, M.A.: Textile robot for matching and pick up clothes based on color recognition. Asian J. Appl. Sci. Technol. (AJAST) 1(3), 62-65 (2017) 
12. Miaskiewicz, T., Kozar, K.A.: Personas and user-centered design: how can personas benefit product design processes? Des. Stud. 32(5), 417-430 (2011). https://doi.org/10.1016/j.des tud.2011.03.003

13. Kane, S.K., Jayant, C., Wobbrock, J.O., Ladner, R.E.: Freedom to roam: a study of mobile device adoption and accessibility for people with visual and motor disabilities. In: Proceedings of the 11th International ACM SIGACCESS Conference on Computers and Accessibility, pp. 115-122. Association for Computing Machinery, New York (2009). https://doi.org/10. $1145 / 1639642.1639663$

14. Law, C.M., Yi, J.S., Choi, Y.S., Jacko, J.A.: Are disability-access guidelines designed for designers? Do they need to be? In: Proceedings of the 18th Australia Conference on ComputerHuman Interaction: Design: Activities, Artefacts and Environments (OZCHI 2006), pp. 357360. Association for Computing Machinery, New York (2006). https://doi.org/10.1145/122 8175.1228244

15. Rainger, P.: Usability and accessibility of personal digital assistants as assistive technologies in education. Learning with mobile devices: research and development. Learning and Skills Development Agency, London, UK, pp. 131-137 (2004)

16. El-Glaly, Y.N., Peruma, A., Krutz, D.E., Hawker, J.S.: Apps for everyone: mobile accessibility learning modules. ACM Inroads 9(2), 30-33 (2018). https://doi.org/10.1145/3182184

17. About AEGIS. http://www.aegis-project.eu. Accessed 13 June 2020

18. Sulmon, N., Slegers, K., Van Isacker, K., Gemou, M., Bekiaris, E.: Using personas to capture assistive technology needs of people with disabilities (2010)

19. LeRouge, C., Ma, J., Sneha, S., Tolle, K.: User profiles and personas in the design and development of consumer health technologies. Int. J. Med. Informatics 82(11), 251-268 (2013). https://doi.org/10.1016/j.ijmedinf.2011.03.006

20. Brulé, E., Jouffrais, C.: Representing children living with visual impairments in the design process: a case study with personae. In: Langdon, P., Lazar, J., Heylighen, A., Dong, H. (eds.) Designing Around People, pp. 23-32. Springer, Cham (2016). https://doi.org/10.1007/9783-319-29498-8_3

21. Kane, S., Hurst, A., Buehler, E., Carrington, P., Williams, M.: Collaboratively designing assistive technology. Interactions 21(2), 78-92 (2014)

22. ATHack. http://assistivetech.mit.edu/events. Accessed 14 June 2020

23. Boston Grand Hack 2019. https://grandhack.mit.edu/boston-2019. Accessed 14 June 2020

24. \#include < girl >'s Assistive Technology Hackathon. https://code.likeagirl.io/include-s-assist ive-technology-hackathon-1ff7d1caf83a. Accessed 14 June 2020

25. Gubin, T.A., et al.: A systems approach to healthcare innovation using the MIT hacking medicine model. Cell Syst. 5(1), 6-10 (2017). https://doi.org/10.1016/j.cels.2017.02.012

26. Watkins, S.M., Dunne, L.E.: Functional Clothing Design: From Sportswear to Spacesuits, 1st edn, pp. 2-29. Fairchild Books, New York (2015)

27. Cook, A.M., Polgar, J.M.: Cook \& Hussey's Assistive Technologies: Principles and Practice, 3rd edn, pp. 34-53. Mosby Elsevier, Maryland Heights (2008)

28. Burgstahler, S., Doe, T.: Disability-related simulations: if, when, and how to use them. Rev. Disabil. Stud. 1(2), 4-17 (2004) 